\title{
Recent Advances in Understanding and Use of Oleofoams
}

\author{
Anne-Laure Fameau ${ }^{1 *}$ and Arnaud Saint-Jalmes ${ }^{2}$ \\ ${ }^{1}$ L'Oréal R\&l, Saint-Ouen, France, ${ }^{2}$ Univ Rennes, CNRS, IPR (Institut de Physique de Rennes) - UMR 6251, Rennes, France
}

\section{OPEN ACCESS}

Edited by:

Luiz Henrique Fasolin,

Campinas State University, Brazil

Reviewed by:

Bernard Paul Binks,

University of Hull, United Kingdom Björn Braunschweig,

University of Münster, Germany

*Correspondence:

Anne-Laure Fameau anne-laure.fameau@rd.loreal.com

Specialty section:

This article was submitted to

Sustainable Food Processing, a section of the journal

Frontiers in Sustainable Food Systems

Received: 03 May 2020

Accepted: 17 June 2020

Published: 06 August 2020

Citation:

Fameau A-L and Saint-Jalmes A

(2020) Recent Advances in

Understanding and Use of Oleofoams.

Front. Sustain. Food Syst. 4:110

doi: $10.3389 /$ fsufs. 2020.00110
This review aims at presenting recent advancements on the understanding of oleofoams for food applications. Edible oleofoams are currently based on heating a vegetable oil solution containing a high-melting point component, which crystallize upon cooling. After aeration (mostly by whipping), crystals adsorb to air bubble surfaces. The remaining crystals in excess in the continuous phase form an oleogel. Due to both the presence of crystals at the interface and in the bulk, the resulting oleofoams exhibits high stability to drainage, coalescence, and disproportionation. The mechanisms leading to the high foam stability are still under investigations to understand and to discriminate between bulk and interfacial rheology effects. The research on edible oleofoams are still scarce in comparison to aqueous food foams even if they present promising applications. In the area of molecular gastronomy, oleofoams are already produced. Oleofoams are a great opportunity for food technologists to develop healthier food products with a low fat content associated to new sensorial properties. However, it is important to better understand these systems in order to help food technologists to use oleofoams and it will contribute to expand this new promising area in food science.

Keywords: oleofoams, oleogel, organogel, whipped oil, crystalline particles, pickering stabilization, interfacial rheology, contact angle

\section{INTRODUCTION}

Liquid foams are complex colloidal systems based on gas bubbles dispersed in a liquid continuous phase containing surface-active components giving rise to the foam formation and stabilization (Cantat et al., 2013). Liquid foams are widely applied in many industries, especially in the food industry. Two different categories of liquid foams exist: aqueous or non-aqueous (Friberg, 2010). Non-aqueous foams based on edible oils represent a new promising emerging field for edible applications, and are commonly called oleofoams (Heymans et al., 2017; Dickinson, 2020). These systems are more difficult to obtain in comparison to aqueous foams due to the lower surface-activity of most edible emulsifiers at the oil-air interface in comparison to air-water interface (Fameau and Saint-Jalmes, 2017). Nevertheless, the fundamental research in this area is tremendously growing since 2015, and the industrial potential of oleofoams is high as novel structuring materials to substitute solid fats (Heymans et al., 2017).

Indeed, in the last few decades, both governmental institutions and consumers asked the food industry to improve the nutritional quality of food products. The food industry need to replace saturated fat by unsaturated fat or by lowering fat content in order to improve nutritional aspects of food products (Patel and Dewettinck, 2016). However, it is impossible to simply replace solid fats by edible oils since it leads to a loss of texture, structure, and mouthfeel, which are provided by the presence of solid fats. Oleofoams could be used by food technologists to create food products with 
reduced fat content in combination with new textures and sensorial properties (Gunes et al., 2017; Chisholm et al., 2018; Gehin-Delval et al., 2019a).

In the area of molecular gastronomy, the production of edible oil foams started even well before the increased interest of researchers for these systems the incorporation of air bubbles in the vegetable oils provides a different mouthfeel and appearance to oil products, which are important attributes for molecular gastronomy. The advantages of oleofoams for food technologists are not only based on the texture and reduced fat content, but also on the fact that oleofoams exhibit very long term stability even above room temperature, and they can be obtained with few or even without any additives (Binks and Marinopoulos, 2017). The enhancement of food shelf-life is mandatory to reduce food waste and to keep food products quality during storage. Oleofoams are based on oil without water, which drastically reduced microbial spoilage and therefore less or no preservatives are needed (Heymans et al., 2017). All these advantages are also very important regarding the increasing trend in developing "clean label" in food industry (Heymans et al., 2017).

This mini-review describe the recent studies and patents on edible oleofoams and summarizes the current state of knowledge concerning the stabilization mechanisms.

\section{OLEOFOAMS: FORMATION, STRUCTURE, AND APPLICATIONS}

Oleofoams are made of gas bubbles in a continuous oil phase (Figure 1). The first discovery of oil foams was done in the 1970s (Sanders, 1970). These systems regained interest more than 30 years later with the studies by Shrestha et al. (2006, 2008, 2010). However, it is only in the last 5 years that this field began to grow tremendously for edible applications (Heymans et al., 2017). Edible oil foams are obtained in two steps. In the first step, an edible vegetable oil containing a high-melting edible component are heated above the solubility boundary of the mixture (Figure 1) (Fameau, 2018). By cooling the mixture below the solubility boundary, the high-melting edible component crystallizes. In the second step, the mixture is whipped to introduce air bubbles leading to oleofoams. The whipping can take place during or after the cooling process (Fameau and Saint-Jalmes, 2017). Whatever the foaming process, foam formation is only possible when crystals are present in solution that is to say below the solubility boundary (Fameau and Saint-Jalmes, 2017). Crystalline particles from various systems have been used to obtain oil foams. The first systems described in the literature for food applications were based on mono and/or diglycerides (Shrestha et al., 2006, 2008, 2010). Then, it is only 10 years later that three new fatty components have also been shown to give oil foams: fatty alcohols (Fameau et al., 2015), fatty acids (Binks et al., 2016), and triglycerides (Mishima et al., 2016). Last year, the mixture of phytosterols and monoglycerides have been demonstrated also to form oleofoams (Truong et al., 2019). Another approach to produce oleofoams is to use oil/fat containing a high proportion of saturated mediumlength fatty acid chains in order to have additive-free edible oleofoams as described in 2017 after the first study on oleofoams based on triglycerides (Binks and Marinopoulos, 2017). By controlling the temperature, the triglycerides with high melting point crystallize and other triglycerides with low melting point remain in a liquid state. Coconut oil, shea butter and cocoa butter are naturally composed of crystalline particles dispersed inside a continuous oil liquid phase giving rise to oil foams (Binks and Marinopoulos, 2017).

The gas bubbles are generally covered by a dense layer of adsorbed crystals that considerably reduces disproportionation and coalescence (Binks et al., 2016). The role of the crystals on the stabilization is described sections Crystal Properties for the Design of Oleofoams and Stabilization Mechanisms. The fraction of non-adsorbed crystals, which increases with crystals concentration, serve to strengthen the gel network in the continuous oil phase (Co and Marangoni, 2012; Mishra et al., 2020). When the excess of crystalline particles in the continuous oil phase is high enough (i.e., above the gel point depending on the temperature), the particles form a network because of short-range (van der Waals) attractive forces between crystals. An oleogel is created which impends buoyancydriven creaming of air bubbles within the foam. Therefore, oleofoams exhibit high stability against drainage, coalescence and disproportionation. Some oleofoam systems are stable during months (Fameau et al., 2015; Binks et al., 2016). The presence of crystalline particles is the key parameter to produce oleofoams (see sections Crystal Properties for the Design of Oleofoams and Stabilization Mechanisms). Each foam bubble is covered by adsorbed crystalline particles, which prevent the relaxation of the bubble to a spherical shape. In oleofoams, most of the bubbles are non-spherical and possess textured surfaces (Binks et al., 2016).

The resulting rheological properties of oleofoams is still debated, but appears to be both due to contribution of the crystals coating the bubbles and to the rheology of the gelled continuous phase in which the bubbles are dispersed (Gunes et al., 2017; Mishra et al., 2020; Saha et al., 2020) (see section Stabilization Mechanisms). Gunes et al. determined that the elastic modulus of oleofoams are two orders of magnitude higher than the modulus of a broken (=highly sheared) oleogel at the same concentration of monoglycerides in the oil, but still lower that the initial unbroken network at rest (Gunes et al., 2017). The whipping/mixing process, by modifying the bulk network probably plays a crucial role, by both setting the morphology of the crystals and the actual rheological properties of the oleofoam continuous phase (Gunes et al., 2017). At low frequencies, some oleofoams tends to behave as viscous liquids (Fameau et al., 2015). Therefore, the oleofoams have a good flowability without collapsing due to their rheological properties (Gunes et al., 2017; Heymans et al., 2018). This is a key parameter for the food industry that need easy-to-handle product that could be pumped through pipes and easy mixed with other components (Heymans et al., 2018). However, the flowable character strongly depends on the amount of incorporated gas, and of the size of the gas pockets. It is important to keep in mind that to produce oleofoams from oleogels, they need to behave as viscous dispersions once the whipping or mixing process starts in order to allow air 


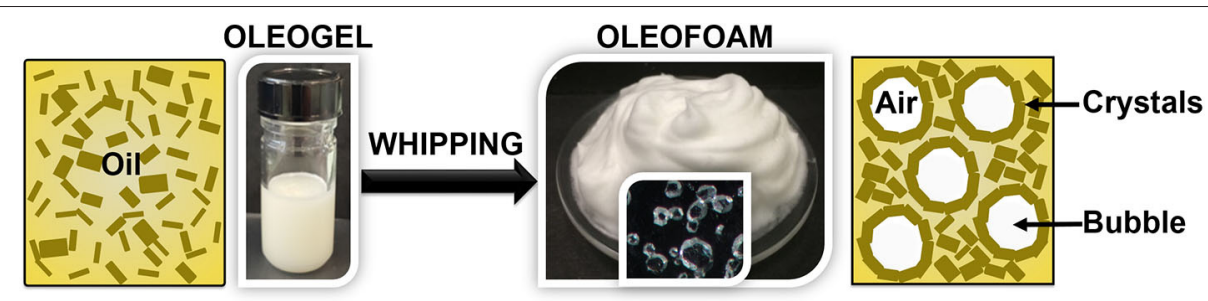

FIGURE 1 | By whipping an oleogel containing crystals inside a liquid oil, oleofoams are obtained. Crystals stabilize the oleofoam due to their presence in bulk and at the interface as observed by optical microscopy.

incorporation, implying specific rheological properties (such as yielding) of these oleogels (Mishra et al., 2020). The gas incorporation process is crucial: it sets the size of the bubbles, the amount of gas, the rheology of the continuous phase and the morphology of the crystals. All these intermediate-scales parameters then control the macroscopic stability and rheology of the produced oleofoam.

In the last 2 years, oleofoams were described in several patents to partially replace fat in food products (Chisholm et al., 2018; Gunes et al., 2018). For example, sponge cakes, biscuits and laminated pastry can be obtained from olefoams by replacing part of the butter (Chisholm et al., 2018; Gehin-Delval et al., 2019a). The oleofoams were based on 10 wt.\% of cacao butter improver (CBI) in high oleic sunflower oil (Chisholm et al., 2018). At $60^{\circ} \mathrm{C}, \mathrm{CBI}$ is soluble in the high oleic sunflower oil. All the food products obtained with oleofoams were very similar to the references based on butter by technical testing. However, the food products with oleofoams contained less fat by volume (Chisholm et al., 2018).

\section{CRYSTAL PROPERTIES FOR THE DESIGN OF OLEOFOAMS}

The key criterion for crystals to adsorb at the air/oil interface is to exhibit a suitable contact angle below $90^{\circ}$ (Binks et al., 2016; Fameau and Saint-Jalmes, 2017). To understand the arrangement of triacylglycerols crystals around the air bubbles of an oleofoam based on salad oil, Mishima and coworkers used synchrotron radiation microbeam X-ray diffraction (Mishima et al., 2016). They demonstrated that the lamellar planes of the crystals near the air-oil surface are arranged almost parallel to the surface (Mishima et al., 2016). It means that the lamellar planes composed of methyl end groups are facing the air phase, whereas the lateral planes composed of glycerol groups are connected to each other through the crystals adsorbed at the air-oil surface (Mishima et al., 2016). We can suppose that the same type of arrangement occurs for the other crystalline particles based on fatty acids and fatty alcohols.

For aqueous foams stabilized by particles called Pickering foams, both the foamability and foam stability depend on the size, shape and concentration of solid particles (Lam et al., 2014). These parameters are also important for oleofoams as highlighted by the first studies (Shrestha et al., 2010; Fameau et al., 2015;
Binks et al., 2016; Gunes et al., 2017). Needle-like and plateletlike shapes lead to adsorption, whereas spherulitic shapes do not allow contact line pinning and hence do not favor adsorption (Gunes et al., 2017; Mishra et al., 2020). To change the size and shape of the crystalline particles, Mishima et al. changed the crystallization and tempering process for a system based on longchain triglycerides dispersed in salad oil (Mishima et al., 2016). The quantity of foam then varied as a function of the process and resulting polymorphs showing the influence of crystals size and morphology. In this study, the best foaming agent was tiny $\beta$ fat crystals (Mishima et al., 2016). Another study from Heymans et al. confirmed the effect of tempering on the foaming properties (Heymans et al., 2018), whereas other authors did not observe a link between foam quantity and crystalline polymorphs (Gunes et al., 2017). Such attempts to correlate crystals size, morphology, and polymorphs with the oleofam properties need to be done with precautions; for instance, tempering also affect the solid fat content and the bulk rheological properties, which have a strong impact on the oleofams (Mishra et al., 2020).

It is also suggested that the size and shape of the particles could be the main parameter governing the bubbles size and not the bulk rheology (Gunes et al., 2017). Gunes et al. showed that to decrease the bubbles size in oleofoams, the size of the crystals need to be decreased. Whipping induced the breakage of the crystals. On the opposite, Mishra et al. showed that the size of the bubbles actually depends on the rheology of the continuous phase: they evidence correlations between the imposed shear rate, the morphology and sizes of the crystals, the yield stress of the solution and the final bubbles size. In particular, the highest bulk yield stresses gave the smallest bubbles. Such apparently different results tend to show that there are still missing parameters, most likely linked to the gas incorporation process that need to be taken into account to identify the real correlations. Indeed, no data exist on the size of the crystals inside the oil foams in comparison to the size of the crystals initially contained in the oleogel. Scattering techniques such as Ultra Small Angle Scattering X-rays (USAXS) and neutrons (USANS) could be helpful to determine the crystals size inside the oleofoams as a function of the whipping process (Peyronel et al., 2014; Mikhailovskaya et al., 2017; Marangoni et al., 2020). Moreover, the structure at the interface, the thickness of the interfacial layer, and the resulting interfacial rheological properties are still unknown. The use of neutron and X-ray reflectivity combined with interfacial rheology could help to obtain new insights on 


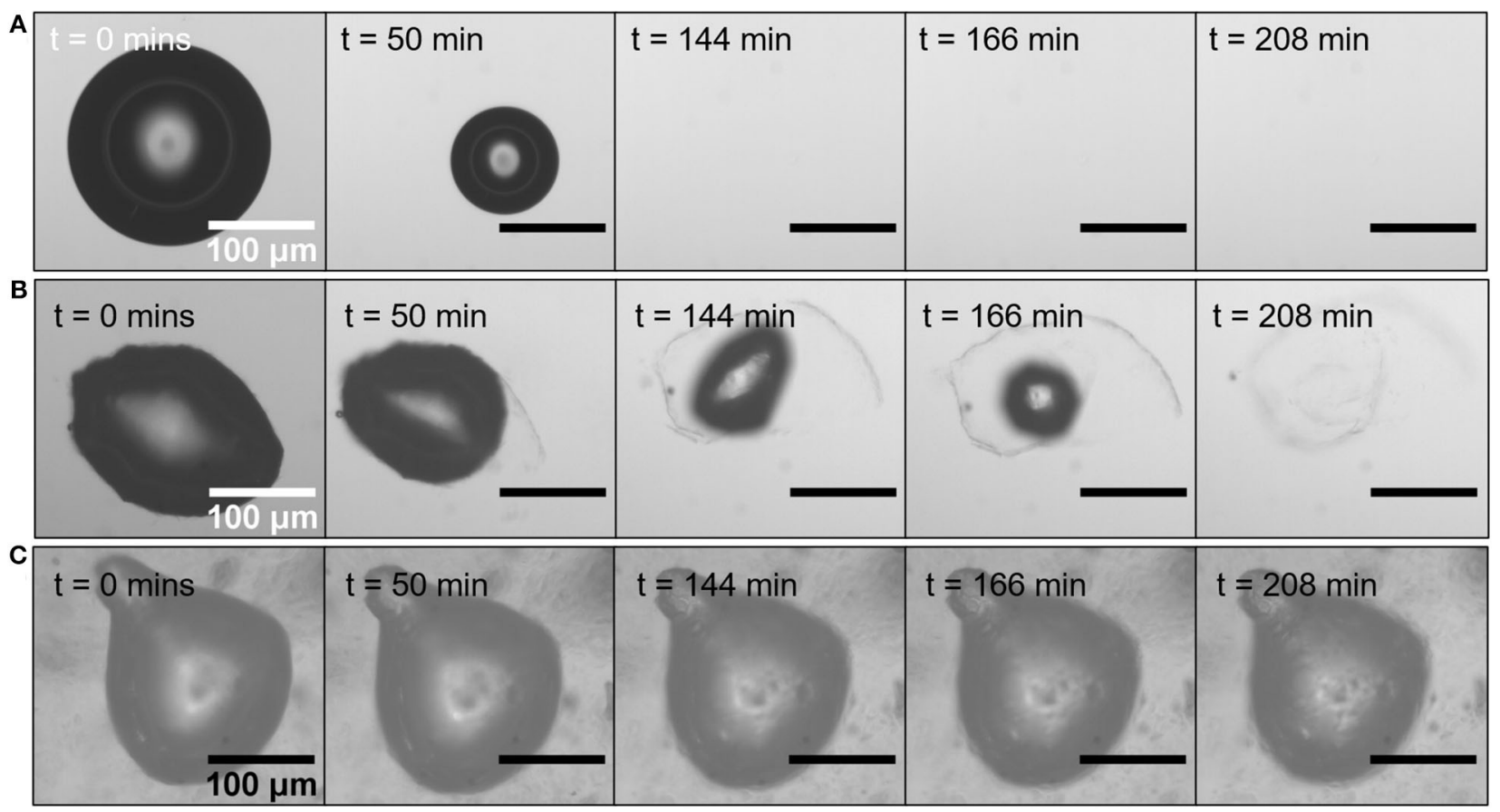

FIGURE 2 | Image sequences of evolution of air bubbles at $25^{\circ} \mathrm{C}$. (A) A bare bubble quickly dissolve in sunflower oil. (B) Bubble coated by synthetic waxes in sunflower oil. The solid interfacial layer unfold leading to bubble dissolution. (C) Bubble inside an oleofoam remains stable over the same timescale. Reproduced from reference (Saha et al., 2020) with permission from Springer

the interfacial properties depending on the nature of the fatty components (Fameau and Salonen, 2014).

\section{STABILIZATION MECHANISMS}

In oleofoams, the long-term stability result from both bulk and interfacial contributions (Gunes et al., 2017; Saha et al., 2020). Various destabilizing phenomena tends to destroy such gas dispersions. It is therefore important to associate the interfacial and bulk properties only to the destabilization mechanism that they actually hinder. The presence of the crystals layer at the bubble surface has a first stabilizing effect by reducing the surface tension, but they act mostly through the occurrence of a packed elastic layer (Shrestha et al., 2006; Saha et al., 2020). The main goal of such a layer is to prevent coalescence and disproportionation (or ripening). By diluting oleofoams with oil, only single bubbles can be observed (Gunes et al., 2017; Saha et al., 2020). The crystals remained attached to the bubbles and the non-spherical shape remained during few minutes to hours depending on the oleofoam systems and process (Figure 2B) (Gunes et al., 2017; Saha et al., 2020). That is to say many orders of magnitude longer that the relaxation timescales of the bare bubble in oil (Figure 2A). However, when crystals coating bubbles are diluted in a high quantity of oil, it was also observed that the gas bubbles dissolve, whereas no dissolution was observed for bubbles in the oleofoam (Figures 2B,C) (Gunes et al., 2017). These first results were confirmed this year by Saha et al., who demonstrated that the crystals were able to leave slowly the interface under a bubble dissolution driving force leading to the disappearance of the bubbles as illustrated in Figure 2 (Saha et al., 2020). The behavior of a bubble embedded in an oleofoam that is to say surrounded by crystals in the bulk is not the same than a bubble surrounded only by liquid oil. Bubbles in an oleofoam owe their stability to both bulk and interfacial rheology, whereas bubbles coated by crystals but surrounded by liquid oil are only stabilized due to the interfacial dilatational rheology of the crystal layer. This is not sufficient to avoid bubbles dissolution. Crystals both at the interface and in the bulk are needed to confer long-term stability and avoid Oswald ripening and bubbles dissolution. Thus, the usual Pickering mechanism of stabilization, found for aqueous foams, is here insufficient to arrest bubble dissolution (Saha et al., 2020). Additional effects coming from the crystals in the continuous phase and the closely packed crystals in foam films can also arise to reduce Oswald ripening (Gunes et al., 2017).

Since the first studies in 2015, it was highlighted that the bulk rheological properties are also very important for foam stabilization; here mostly for acting against the gravitational drainage and the coalescence. However, this year it was demonstrated that for yield stresses higher than gravitational ones, no phase separation occurs, as well as no flow within films separating bubbles (Mishra et al., 2020). Oppositely, for very weak gel, a high rate of coalescence with the free surface during whipping is observed, which prevents to wrap bubbles with crystals (Gunes et al., 2017). Without a sufficient contribution of the bulk, the foam stabilization against destabilization mechanism is impossible (Mishra et al., 2020). The interfacial 
contribution on the foam stability is a key parameter, but to obtain stable oleofoams over long timescales, films and bulk contributions are also required. The main problems to date to draw clear conclusions on the stabilization mechanisms come from the fact that in each study the fatty component to stabilize the oil foams was different (waxes, fatty alcohol, fatty acids, mono-glyceride, di-glyceride, and tri-glycerides), as well as their purity, and also the process to produce the foam varied. More fundamental studies on the same systems are needed by combining techniques at different length scales as it was done in the past for aqueous foams (Fameau and Salonen, 2014). The readers need also to keep in mind that the crystallization mechanisms in bulk and at the interface depends on the nature of the fatty components, which are linked to their chemical structure, the nature of the edible liquid phase and the process applied to the systems (cooling rate, shearing rate, etc.).

\section{NEW EDIBLE OIL SYSTEM BASED ON OLEOFOAMS}

A novel application of oleofoams is the creation of air-in-oil-inwater $(\mathrm{A} / \mathrm{O} / \mathrm{W})$ systems, in order to design new food products (Brun et al., 2015; Gehin-Delval et al., 2019b; Goibier et al., 2019). In a first step, an oleofoam is produced. In a second step, the oleofoams is emulsified in a continuous phase containing a food grade emulsifier to stabilize the oil droplets and a food grade thickener to protect against creaming of the droplets. Two examples of $\mathrm{A} / \mathrm{O} / \mathrm{W}$ are described in the literature and highlighted the parameters to take into account to produce A/O/W systems (Brun et al., 2015; Goibier et al., 2019). The solid fat content of the oleogel used to produce the initial oleofoams is one of the crucial parameter. The crystals quantity needs to be high enough to stabilize efficiently the air bubbles, but not too high, otherwise it is impossible to maintain a fluid-like oil phase fluid during the emulsification step. The imposed shear rate is also an important parameter to produce $\mathrm{A} / \mathrm{O} / \mathrm{W}$. Too low shear rates could not cause droplets break-up inside the aqueous phase, but too high shear rates induced release of the air bubbles. These A/O/W system based on oleofoams are already described in a recent patent, in which a standard mayonnaise recipe was prepared by replacing the oil phase of the mayonnaise

\section{REFERENCES}

Binks, B. P., Garvey, E. J., and Vieira, J. (2016). Whipped oil stabilised by surfactant crystals. Chem. Sci. 7, 2621-2632. doi: 10.1039/C6SC00046K

Binks, B. P., and Marinopoulos, I. (2017). Ultra-stable self-foaming oils. Food Res. Int. 95, 28-37. doi: 10.1016/j.foodres.2017.02.020

Brun, M., Delample, M., Harte, E., Lecomte, S., and Leal-Calderon, F. (2015). Stabilization of air bubbles in oil by surfactant crystals: a route to produce air-in-oil foams and air-in-oil-in-water emulsions. Food Res. Int. 67, 366-375. doi: 10.1016/j.foodres.2014.11.044

Cantat, I., Cohen-Addad, S., Elias, F., Graner, F., Höhler, R., Pitois, O., et al. (2013). Foams: Structure and Dynamics. Oxford: OUP. doi: 10.1093/acprof:oso/9780199662890.001.0001 by the oleofoam (10 wt.\% of CBI inside high oleic sunflower oil) (Gehin-Delval et al., 2019b). The resulting mayonnaise based on oleofams was less greasy and had a lower density than the standard mayonnaise based only on high oleic sunflower oil. As consumers, generally add mayonnaise to their meal by volume and not by weight, the mayonnaise based on oleofam enable to reduce the fat consumed and to deduce the consumption of saturated fat.

\section{CONCLUDING REMARKS}

Oleofoams are very promising for the food industry since the benefits include long-term stability above room temperature, healthier food products with a low fat content, and new sensorial properties. In the recent years, the main building blocks explaining the oleofoams stability have been identified, allowing us to draw of first picture based on contributions from interfaces and bulk. The crystals govern the entire foam stability. However, a deeper understanding on the stabilization mechanisms is still required to help food industry to design new food products based on oleofoams. In the future, it will be important to continue the recent works to establish the detailed link between the rheological properties of the oleogel and the ones of the resulting oleofoams, and the crystal concentration, their size and shape, through the foaming process (Mishra et al., 2020). It is important also to separate bulk and interfacial effects. A better understanding of oleofoams could be obtained by using techniques and modifying some technical approaches developed to study Pickering aqueous foams and emulsions, but also oleogels and lipid crystallization such as: interfacial rheology, NMR, microscopy techniques, neutron, and X-ray scattering techniques (Fameau and Salonen, 2014; Mikhailovskaya et al., 2017; Marangoni and Garti, 2018; Low et al., 2020; Metilli et al., 2020). New fundamental insights will offer the possibility to design new food products, but also oil foams for cosmetic and pharmaceutical applications.

\section{AUTHOR CONTRIBUTIONS}

A-LF was the main author of the manuscript. AS-J contributed in the manuscript writing and assisting the main author. All authors contributed to the article and approved the submitted version. 
Fameau, A.-L., Lam, S., Arnould, A., Gaillard, C., Velev, O. D., and Saint-Jalmes, A. (2015). Smart nonaqueous foams from lipid-based oleogel. Langmuir 31, 13501-13510. doi: 10.1021/acs.langmuir.5b03660

Fameau, A.-L., and Saint-Jalmes, A. (2017). Non-aqueous foams: Current understanding on the formation and stability mechanisms. Adv. Colloid Interface Sci. 247, 454-464. doi: 10.1016/j.cis.2017.02.007

Fameau, A.-L., and Salonen, A. (2014). Effect of particles and aggregated structures on the foam stability and aging. Comptes Rendus Phys. 15, 748-760. doi: 10.1016/j.crhy.2014.09.009

Friberg, S. E. (2010). Foams from non-aqueous systems. Curr. Opin. Colloid Interface Sci. 15, 359-364. doi: 10.1016/j.cocis.2010.05.011

Gehin-Delval, C., Chisholm, H., Chung, W., Deyber, H., Destribats, M. J., Gunes, Z. D., et al. (2019a). Method for Forming a Laminated Pastry. Patent No. US 2019/0200625 A1, Vervey.

Gehin-Delval, C., Chisholm, H., Gunes, Z. D., Deyber, H., Pelloux, C., Schafer, O., et al. (2019b). Food Composition Comprising Gas Bubbles. Patent No. US 10, 383, 352 B2, Vervey.

Goibier, L., Pillement, C., Monteil, J., Faure, C., and Leal-Calderon, F. (2019). Emulsification of non-aqueous foams stabilized by fat crystals: Towards novel air-in-oil-in-water food colloids. Food Chem. 293, 49-56. doi: 10.1016/j.foodchem.2019.04.080

Gunes, D. Z., Murith, M., Godefroid, J., Pelloux, C., Deyber, H., Schafer, O., et al. (2017). Oleofoams: properties of crystal-coated bubbles from whipped oleogels-evidence for pickering stabilization. Langmuir 33, 1563-1575. doi: 10.1021/acs.langmuir.6b04141

Gunes, Z. D., Schafer, O., Chisholm, H., Deyber, H., Pelloux, C., and Binks, B. P. (2018). Lipid Based Foam. Patent No.WO 2016/150978, Vervey.

Heymans, R., Tavernier, I., Danthine, S., Rimaux, T., Van der Meeren, P., and Dewettinck, K. (2018). Food-grade monoglyceride oil foams: the effect of tempering on foamability, foam stability and rheological properties. Food Funct. 9, 3143-3154. doi: 10.1039/C8FO00536B

Heymans, R., Tavernier, I., Dewettinck, K., and Van der Meeren, P. (2017). Crystal stabilization of edible oil foams. Trends food Sci. Technol. 69, 13-24. doi: 10.1016/j.tifs.2017.08.015

Lam, S., Velikov, K. P., and Velev, O. D. (2014). Pickering stabilization of foams and emulsions with particles of biological origin. Curr. Opin. Colloid Interface Sci. 19, 490-500. doi: 10.1016/j.cocis.2014.07.003

Low, L. E., Siva, S. P., Ho, Y. K., Chan, E. S., and Tey, B. T. (2020). Recent advances of characterization techniques for the formation, physical properties and stability of Pickering emulsion. Adv. Colloid Interface Sci. 277:102117. doi: $10.1016 /$ j.cis.2020.102117

Marangoni, A. G., and Garti, N. (eds.). (2018). Edible Oleogels: Structure and Health Implications. San Diego, CA: AOC Press.

Marangoni, A. G., Van Duynhoven, J. P. M., Acevedo, N. C., Nicholson, R. A., and Patel, A. R. (2020). Advances in our understanding of the structure and functionality of edible fats and fat mimetics. Soft Matter 16, 289-306. doi: 10.1039/C9SM01704F

Metilli, L., Francis, M., Povey, M., Lazidis, A., Marty-Terrade, S., Ray, J., et al. (2020). Latest advances in imaging techniques for characterizing soft, multiphasic food materials. Adv. Colloid Interface Sci. 279:102154. doi: 10.1016/j.cis.2020.102154
Mikhailovskaya, A., Zhang, L., Cousin, F., Boue, F., Yazhgur, P., Muller, F., et al. (2017). Probing foam with neutrons. Adv. Colloid Interface Sci. 247, 444-453. doi: 10.1016/j.cis.2017.07.024

Mishima, S., Suzuki, A., Sato, K., and Ueno, S. (2016). Formation and microstructures of whipped oils composed of vegetable oils and high-melting fat crystals. J. Am. Oil Chem. Soc. 93, 1453-1466. doi: 10.1007/s11746-016-2888-4

Mishra, K., Dufour, D., and Windhab, E. J. (2020). Yield stress dependent foaming of edible crystal-melt suspensions. Cryst. Growth Des. 20, 1292-1301. doi: 10.1021/acs.cgd.9b01558

Patel, A. R., and Dewettinck, K. (2016). Edible oil structuring: an overview and recent updates. Food Funct. 7, 20-29. doi: 10.1039/C5FO0 1006C

Peyronel, F., Pink, D. A., and Marangoni, A. G. (2014). Triglyceride nanocrystal aggregation into polycrystalline colloidal networks: Ultrasmall angle X-ray scattering, models and computer simulation. Curr. Opin. Colloid Interface Sci. 19, 459-470. doi: 10.1016/j.cocis.2014. 07.001

Saha, S., Saint-Michel, B., Leynes, V., Binks, B. P., and Garbin, V. (2020). Stability of bubbles in wax-based oleofoams: decoupling the effects of bulk oleogel rheology and interfacial rheology. Rheol. Acta 59, 255-266. doi: 10.1007/s00397-020-01192-x

Sanders, P. A. (1970). Stabilization of aerosol emulsions and foams. J. Soc. Cosmet. Chem. 21, 377-391.

Shrestha, L. K., Aramaki, K., Kato, H., Takase, Y., and Kunieda, H. (2006). Foaming properties of monoglycerol fatty acid esters in nonpolar oil systems. Langmuir 22, 8337-8345. doi: 10.1021/la061204h

Shrestha, L. K., Shrestha, R. G., Sharma, S. C., and Aramaki, K. (2008). Stabilization of nonaqueous foam with lamellar liquid crystal particles in diglycerol monolaurate/olive oil system. J. Colloid Interface Sci. 328, 172-179. doi: 10.1016/j.jcis.2008.08.051

Shrestha, R. G., Shrestha, L. K., Solans, C., Gonzalez, C., and Aramaki, K. (2010). Nonaqueous foam with outstanding stability in diglycerol monomyristate/olive oil system. Colloids Surfaces A Physicochem. Eng. Asp. 353, 157-165. doi: 10.1016/j.colsurfa.2009.11.007

Truong, T., Prakash, S., and Bhandari, B. (2019). Effects of crystallisation of native phytosterols and monoacylglycerols on foaming properties of whipped oleogels. Food Chem. 285, 86-93. doi: 10.1016/j.foodchem.2019. 01.134

Conflict of Interest: The authors declare that the research was conducted in the absence of any commercial or financial relationships that could be construed as a potential conflict of interest.

Copyright (C) 2020 Fameau and Saint-Jalmes. This is an open-access article distributed under the terms of the Creative Commons Attribution License (CC BY). The use, distribution or reproduction in other forums is permitted, provided the original author(s) and the copyright owner(s) are credited and that the original publication in this journal is cited, in accordance with accepted academic practice. No use, distribution or reproduction is permitted which does not comply with these terms. 